A N N A L E S Annales de Bretagne et des Pays de l'Ouest

\title{
Justice et émotions sous l'Ancien Régime
}

Histoire sensible des séparations conjugales (Poitou, $\mathrm{XVII}^{\mathrm{e}}$-XVIII ${ }^{\mathrm{e}}$ siècles)

\section{Gwénael Murphy}

\section{OpenEdition}

\section{Journals}

Édition électronique

URL : http://journals.openedition.org/abpo/3841

DOI : 10.4000/abpo.3841

ISSN : 2108-6443

Éditeur

Presses universitaires de Rennes

\section{Édition imprimée}

Date de publication : 12 juillet 2018

Pagination : 107-121

ISBN : 978-2-7535-7604-9

ISSN : 0399-0826

Référence électronique

Gwénael Murphy, "Justice et émotions sous l'Ancien Régime », Annales de Bretagne et des Pays de l'Ouest [En ligne], 125-2 | 2018, mis en ligne le 12 juillet 2020, consulté le 05 janvier 2021. URL : http:// journals.openedition.org/abpo/3841 ; DOI : https://doi.org/10.4000/abpo.3841 


\title{
Justice et émotions sous l'Ancien Régime Histoire sensible des séparations conjugales (Poitou, XVII - -XVIII ${ }^{\mathrm{e}}$ siècles)
}

\author{
Gwénael MuRPHY \\ Professeur agrégé à l'université de la Nouvelle-Calédonie, \\ docteur en histoire (EHESS, Paris) - TROCA ("Trajectoires d'Océanie ", \\ équipe émergente en lettres, langues et sciences humaines à l'université de Nouméa)
}

Les années 2000 furent marquées par l'émergence des " émotions " en tant qu'objet d'analyse critique. Si des précurseurs en ce domaine ne sauraient être oubliés, en particulier Alain Corbin, les interrogations sur les émotions investissent le champ médiatique de façon inédite au tournant de cette décennie. L'une des principales critiques adressées aux décideurs politiques serait alors de céder, pour reprendre une expression convenue, à la " dictature de l'émotion ", chaque fait divers engendrant une nouvelle loi $^{1}$. Les juristes tentent de mettre en perspective le rôle de ces émotions dans les processus de régulation sociale, alors que les sciences humaines se saisissent, massivement, de ce " continent qui émerge " pour reprendre l'expression de Piroska $\mathrm{Nagy}^{2}$. Dans la foulée des neurosciences, mais aussi en lien avec la demande sociale autour des émotions, psychologues, sociologues, anthropologues et historiens ouvrent de nombreuses perspectives autour de ce nouveau paradigme. Si les travaux des historiens concernent en majorité le XIXe siècle, l'époque médiévale est rapidement interrogée par Damien Boquet et Piroska Nagy, dans la suite des pistes proposées

1. Dans une bibliographie très abondante sur cette "dictature ", voir l'article de CARON, Thierry, Le Monde, 26 janvier 2011 en ligne : [http://www.lemonde.fr/idees/chronique/2011/01/26/dictature-de-l-emotion_1470593_3232.html].

2. NAGY, Piroska, "Faire l'histoire des émotions à l'heure des sciences émotions ", BUCEMA, hors-série $\mathrm{n}^{\circ}$ 5, 2013 ([https://cem.revues.org/12539]). Ces interrogations des juristes sont synthétisées, entre autres, par HAMMER, Raphäl, Droit et émotions dans le processus de régulation juridique et sociale, Genève, Centre d'étude, de technique et d'évaluation législative, 2010, 201 p. Voir également, pour une vision nord-américaine, l'article du sociologue TURNER, Jonathan H., "Justice and Emotions ", Social Research Justice, vol. 20 , sept. 2007, p. 288-311. 
par Barbara H. Rosenwein ${ }^{3}$. Les années 2010 offrent aux historiens les premières interrogations épistémologiques sur l'objet "émotions " ainsi qu'une synthèse monumentale qui rassemble les travaux d'une quarantaine de chercheurs, en $2016^{4}$.

La contribution proposée s'inscrit dans la lignée de cette historiographie des affects, avec le souhait de tenter une histoire sensible des séparations conjugales au croisement des préoccupations des historien(ne)s du droit et de ceux et celles de la justice ${ }^{5}$. La perspective de genre sous-tend, bien entendu, cette analyse. La difficulté du contexte législatif sera présentée en premier lieu, avant que nous nous attachions au lien entre expression judiciaire des émotions et société d'ordres sous l'Ancien Régime. Nous tenterons ensuite d'élaborer une lexicologie de celles-ci dans le cadre des procédures de séparations conjugales, avant de proposer des pistes de réflexion sur leurs usages et leur instrumentalisation, en particulier lorsqu'il s'agit pour un époux ou une épouse de défendre une réputation menacée.

\section{Exprimer ses émotions dans les procédures de séparation : une impossibilité juridique?}

Afin de capter les moments de fortes tensions, physiques, morales ou verbales, dans les couples aux XVII ${ }^{\mathrm{e}}$ et XVIII ${ }^{\mathrm{e}}$ siècles, sans omettre le fait que les sources imprimées, iconographiques ou littéraires peuvent représenter une approche possible, nous avons mis l'accent sur les archives judiciaires, particulièrement les procédures de séparations conjugales. Celles-ci présentent l'avantage de retranscrire les dépositions des plaignant(e)s en s'appuyant sur leurs propres mots, même s'il convient de garder à l'esprit qu'ils sont rapportés par un scribe, le greffier, qui peut " aplanir " les émotions lorsqu'il les reporte dans les procès-verbaux. Le registre lexical usuel de ces procédures, nous le verrons, rejette pendant longtemps le vocabulaire émotionnel ${ }^{6}$.

3. Rosenwein, Barbara H., "Histoire de l'émotion : méthodes et approches ", Cahier de civilisation médiévale, $\mathrm{n}^{\circ}$ 193, vol. 49, 2006, p. 33-48; BoquET, Damien et PIROSKA, Nagy, Sensible Moyen-Âge. Une histoire des émotions dans l'Occident médiéval, Paris, Seuil, 2015, $480 \mathrm{p}$.

4. Deluermoz, Quentin, Fureix, Emmanuel, Mazurel, Hervé et OuAlde, M'hamed, «Écrire l'histoire des émotions : de l'objet à la catégorie d'analyse ", Revue d'Histoire du XIXe siècle, $n^{\circ} 47,2013$, p. 155-189. Pour la synthèse : CoRBIn, Alain, CourTine, Jean-Jacques et Vigarello, Georges (dir.), Histoire des émotions, Paris, Seuil, 2016, 3 vol.

5. Les travaux de Frédéric CHAUVAUD permettent de nombreuses approches, sur le $\mathrm{XIX}^{\mathrm{e}}$ siècle en particulier, de l'étude des émotions à travers les archives judiciaires (Histoire de la haine : une passion funeste, Rennes, Presses universitaires de Rennes, 2014, 412 p.; les actes du colloque codirigé avec PRÉTOU Pierre, Clameurs publiques et émotions judiciaires de l'Antiquité à nos jours, Rennes, PUR, 2014, 320 p.). Il convient également de citer, en histoire médiévale, VERDON, Louise, "Émotions, justice et normes : la fabrique du lien social ", Critiques, vol. 63, 2007, p. 47-57 et son ouvrage La voix des dominés. Communautés et seigneuries en Provence au bas Moyen-Âge, Rennes, PUR, 2013, 224 p.

6. Concernant l'usage des archives judiciaires, voir GARnOT, Benoît (dir.), La justice et l'histoire. Sources judiciaires à l'époque moderne, Paris, Bréal, 2006, 288 p. Parmi les 
Les séparations prononcées par les justices d'Ancien Régime peuvent être de biens, et dans ce cas n'intervenir que sur le plan économique en provoquant la dissolution de la communauté, ou de corps, dispensant les conjoints de la vie commune à la suite de violences subies par l'épouse et mettant sa vie en danger ${ }^{7}$. Plus rarement apparaissent les procès en adultère ou en impuissance ${ }^{8}$. Ces archives laissent fréquemment apparaître, de manière explicite, les violences conjugales, suggérant la présence du registre des émotions ${ }^{9}$. Nous sommes dans le champ du privé, ou à sa proximité, et la haine, la colère, l'amertume, la honte ou la peur se doivent d'être exposées, voire instrumentalisées, ainsi que l'a étudié Robert Muchembled, afin d'obtenir gain de cause auprès de la juridiction compétente ${ }^{10}$.

Plus d'un millier de procédures de séparations conjugales sont recensées à ce jour dans la province du Poitou ${ }^{11}$. Si elles se retrouvent essentiellement dans les justices civiles, seigneuriales en particulier, ainsi qu'en

publications de Benoît Garnot sur l'histoire de la justice, on consultera avec profit son analyse des factums en tant que sources dans son ouvrage Questions de justice, 1667-1789, Paris, Belin, 2006, 159 p.

7. Voir l'étude de LANDELLE, Marie, "Les plaintes en séparation sont éternelles » : la séparation de biens dans la haute société parisienne au milieu du XVIII siècle, 1730-1761, thèse pour le diplôme d'archiviste paléographe, Paris, École nationale des chartes, 2012.

8. Concernant l'adultère, nous avons repéré une seule procédure en Poitou (Arch. dép. de la Vienne, F-20 : mémoire pour maître Frouin de la Godinière, de Thouars, contre son épouse pour adultère [1767], la procédure civile se trouve en 1B1-835). Voir FARGE, Arlette, Un ruban et des larmes. Un procès pour adultère au XVIII siècle, Paris, Éditions des Busclats, 2011, 80 p. et WaLCH, Agnès, Histoire de l'adultère, XVIe-XIXe siècles, Paris, Perrin, 2009, 420 p. Sur l'impuissance, Darmon, Pierre, Le tribunal de l'impuissance. Virilité et défaillances conjugales dans l'ancienne France, Paris, Seuil 1979, 311 p.

9. Les violences conjugales en France à l'époque moderne restent peu étudiées sur le plan historiographique. On consultera avec profit les travaux de : BEAUVALET-BOUTOUYRIE, Scarlett, La solitude, XVII -XVIII $^{e}$ siècles, Paris, Belin, 2008, p. 79-89; FARGE, Arlette et FoucaulT, Michel, Le désordre des familles, Paris, Gallimard, 1982, 354 p.; FARGE, Arlette, La vie fragile. Violences, pouvoirs et solidarités à Paris au XVII siècle, Paris, Hachette, 1986, p. 101-122; GODINEAu, Dominique, Les femmes dans la société française, $16^{e}-18^{e}$ siècles, Paris, A. Colin, 2003, p. 41-43; JaRnoux, Philippe, Moi, Hypolithe Radegonde Loz. Un "divorce " au siècle des Lumières, Rennes, Apogée, 2001, 192 p.; MallLaRD, Brigitte, « Les désordres conjugaux en ville à la fin de l'Ancien Régime " dans Regards sur les sociétés modernes. Mélanges offerts à Claude Petitfrère, Tours, Presses universitaires de Tours, 1997, p. 411-420; NOLDE, Dorothea, Le meurtre du conjoint. Pouvoir et violence dans le mariage à l'époque moderne, Cologne, Boehlau, 2003.

10. MuCHEMBLED, Robert, Une histoire de la violence, Paris, Seuil, 2008, 498 p.

11. Le corpus est constitué par le dépouillement de $20 \%$ des archives judiciaires de la province du Poitou au XvIII ${ }^{\mathrm{e}}$ siècle, soit plus de deux mille références archivistiques dans les fonds des archives départementales de la Vienne et des Deux-Sèvres, dans le cadre de la préparation d'un ouvrage sur les " désordres conjugaux " sous l'Ancien régime. Les sources sont issues, en majorité, des archives de la justice civile (série 2B des Arch. dép. de la Vienne), criminelle (série 1B), des justices seigneuriales (série 9B ainsi que les séries $B$ et $2 B$ des Arch. dép. des Deux-Sèvres) et enfin des " Papiers du Roi " constitués par les recours auprès du Parlement de Paris (série 8B des Arch. dép. de la Vienne). Les factums recensés en sous-série F-20 et F-21 des Arch. dép. de la Vienne ainsi que les procès en impuissance retrouvés dans la série $\mathrm{G}$ regroupant les documents de la Cour métropolitaine de Bordeaux pour le bureau de Poitiers complètent ce panorama. 
procédure d'appel au présidial, les juridictions criminelle et ecclésiastique ne sont pas écartées.

Cette analyse du lexique émotionnel qui retrace la vie des "mauvais ménages ", selon l'expression consacrée sous la plume des greffiers, débute dans les années 1660. En effet, comme la séparation était avant tout une affaire de droit privé, elle relevait en premier lieu de la coutume. Lointain héritage du Corpus juris civilis romain, la procédure apparaît timidement à partir du XII ${ }^{\mathrm{e}}$ siècle dans les officialités ecclésiastiques. En 1580, la coutume de Paris admet la séparation de biens dans son champ de compétences, puis des arrêts de cours souveraines émergent progressivement, parfois suite à un désistement de la juridiction ecclésiastique, comme à Châlons en $1663^{12}$. Cette évolution des préoccupations de l'Église française semble liée à l'émergence d'une politique de contrôle moral des prêtres par les officialités, dans le sillage de la Contre-Réforme, de la mise en place des séminaires au cours du XVII ${ }^{\mathrm{e}}$ siècle et du renforcement des enquêtes sur les "bonnes pratiques " cléricales. En conséquence, la justice ecclésiastique se dessaisit progressivement de ses prérogatives concernant la vie conjugale au profit des justices civiles, pour se concentrer sur la " police interne " de ses membres. Au siècle suivant, tandis que les procédures de séparation sont sécularisées, Louis Sébastien Mercier déplore la facilité avec laquelle les époux se séparent :

"Le mariage est indissoluble; le divorce est défendu par les lois divines et humaines, mais si deux époux veulent se séparer ils n'ont qu'à se donner des chiquenaudes devant deux témoins, la justice les sépare à l'instant; ils ne peuvent cependant pas se marier à d'autres, mais ils vivent librement, en attendant que la mort leur ait fait l'amitié de limer cette chaine maudite que la déraison leur a rendu si pesante ${ }^{13}$."

Un nouveau droit matrimonial s'élabore avec des fondements solides, diffusé dans de nombreux traités et répertoires de jurisprudence à partir des années $1690^{14}$. La doctrine, issue du principe romain de l'exactio dotis, est stricte : seules des raisons d'ordre financier sont admises pour obtenir une séparation, la dot de l'épouse doit être mise en danger par la mauvaise gestion du mari. Afin d'éviter des "recours abusifs ", les motifs affectifs sont volontairement bannis. L'expression des émotions s'avère de fait presque impossible dans un cadre aussi étroit même s'il est perceptible dans les procédures que certaines femmes usent de

12. Arrêt qui constitue le point de départ de l'étude de LotTin, Alain (dir.), La désunion du couple sous l'Ancien régime. L'exemple du Nord, Lille, Presses universitaires de Lille, 1975, p. 12. Sur l'aspect juridique, voir BART, Jean, Histoire du droit privé de la chute de l'Empire romain au XIX siècle, Paris, Montchrestien, 1998, p. 286-337; CASTALDO, André, Introduction historique au droit, Paris, Dalloz 1998, p. 403-409; Peтот, Pierre, La famille, Paris, Loysel, 1992, p. 450-460; VALDRINI, Patrick, Droit canonique, Paris, Dalloz, 1999, p. 351-357.

13. Mercier, Louis Sébastien, Le Tableau de Paris, Paris, La Découverte, 1982, p. 227-228.

14. Sur cette judiciarisation, voir RÉGINA, Christophe, "L'intrusion de la justice dans les foyers. La violence conjugale jugée devant la sénéchaussée de Marseille au XVIII ${ }^{\mathrm{e}}$ siècle ", Annales de Démographie Historique, nº 2009/2, p. 53-75. 
ce stratagème pour justifier la dissolution de la communauté avec un homme violent.

La conséquence, pour l'historien, est directe : dans notre corpus, seules $5 \%$ des procédures offrent des termes qui relèvent du registre émotionnel dans les diverses dépositions ou actions de défense en justice, soit 48 affaires recensées dans la juridiction du présidial de Poitiers. Les débats qui opposent partisans et détracteurs du droit au divorce au XVIII ${ }^{\mathrm{e}}$ siècle accompagnent l'augmentation des mentions de violences conjugales dans les dépositions, qui ouvrent généralement le champ lexical des émotions ${ }^{15}$. La première déposante concernée par ce corpus se nomme Marguerite David, mariée au cabaretier Louis Bodin, de Varennes, un village du Poitou protestant $^{16}$. En mai 1679, elle sollicite la séparation de cet homme qui passerait beaucoup de temps à boire, jouer, chercher querelle et pratiquer des relations sexuelles extra-conjugales tout en la frappant régulièrement. Malgré cela, précise le greffier, Marguerite entame la procédure " avec beaucoup de tristesse et de déplaisir ". Ces mots, peut-être liés à des sentiments d'ordre religieux, semblent exprimer le regret de l'épouse, une forme de compassion et de compréhension pour le mari. Ils permettent d'appréhender la difficulté d'interprétation, pour l'historien, de l'expression des affects dans les archives judiciaires.

\section{De l'absence d'émotion parmi les femmes du peuple...}

Décrypter les sentiments, le ressenti, la réception des violences par les victimes, leurs bourreaux et les témoins de ces actes à travers des archives judiciaires demeure délicat en raison même du prisme normatif imposé par les documents et leurs rédacteurs. Les mêmes mots se retrouvent d'une justice seigneuriale à une autre, d'un greffier à l'autre sur toute l'étendue de la province du Poitou : les hommes sont des " dissipateurs", qui font de " mauvais marchés ", se livrent à de nombreuses " débauches et jeux " dans

15. En amont, l'étude s'arrête à la révolution judiciaire de 1790 expliquée par CHAUvAUD, Frédéric, PetiT, Jacques Guy et Yvorel, Jean-Jacques, Histoire de la justice de la Révolution à nos jours, Rennes, PUR, 2007, p. 29 : la création en octobre 1790 des justices de paix fait disparaître, de fait, les justices seigneuriales, symboles de l'Ancien Régime.

16. Arch. dép. des Deux-Sèvres, 2B-42 : prévôté royale de Melle, enquêtes civiles (16511685). Il convient de souligner que dans la région où les conversions au protestantisme furent les plus nombreuses en Poitou, autour de Melle, les demandes de séparation représentent près de 10 \% des affaires traitées par la prévôté, soit cinq à dix fois plus que dans le reste de la province. D'une manière plus générale, le Poitou se caractérise par la présence d'une minorité protestante importante dès le XVI ${ }^{\mathrm{e}}$ siècle (voir MARCADE, Jacques, Protestants poitevins de la Révocation à la Révolution, La Crèche, Geste éditions, 1998, 239 p.) concentrée autour d'un réseau de villes moyennes telles Thouars, Loudun, Châtellerault, Parthenay, Lusignan, Melle ou Saint-Maixent. Nos recherches montrent le lien direct entre cette présence protestante et le nombre de demandes en séparation de biens dans les archives judiciaires de la province aux XVII ${ }^{\mathrm{e}}$ et XVIII ${ }^{\mathrm{e}}$ siècles, de l'ordre d'un à dix avec les zones catholiques de la région. Voir notre article " Justice et séparations conjugales sous l'Ancien régime dans le Poitou ", Bulletin de la Société historique et scientifique des Deux-Sèvres, 2013, 4e série, no 9, p. 42-72. 
lesquels ils consomment " journellement les biens de leur communauté ". Une multitude de témoignages recueillis par les magistrats poitevins aux $\mathrm{XVII}^{\mathrm{e}}$ et $\mathrm{XVIII}{ }^{\mathrm{e}}$ siècles peut se résumer à travers les deux dépositions effectuées, par exemple, à l'encontre de René Babin. Ce marchand du bourg de Fors, au sud de Niort, subit une procédure de séparation de la part de son épouse en 1707, après douze années de mariage. Le premier témoin, René Archambault, autre marchand, explique que « ledit Babin va très souvent au cabaret où il laisse de nombreuses dépenses, beaucoup d'argent, mais que là où il dissipe le plus de ce qu'il peut avoir de biens c'est par les mauvais marchés qu'il fait achetant toujours plus qu'il ne faut ". Michel Béraud, pour sa part, explique ensuite « qu'il a reçu très souvent ledit Babin au cabaret où il faisait de forts grandes débauches et de très mauvais marchés, achetant fort cher à crédit où il ne pouvait que perdre considérablement ${ }^{17}$ ". De telles déclarations forment véritablement les stéréotypes dans le domaine des demandes de séparation.

Nul doute que les greffiers aplanissent les émotions exprimées par les épouses. Le fait est marquant : même dans les cas de sévices corporels graves et d'injures, les dépositions se présentent comme de simples procès-verbaux relatant uniquement les faits répréhensibles. Le cas de Marie Aluchaud, mariée au laboureur Sautereau de La Mothe-Saint-Héray, s'avère symptomatique de cette pratique. La déposition relate qu'en 1772 et 1773 elle fut " congédiée de sa maison, son mari a voulu l'empoisonner à deux reprises ", puis au moment de la récolte des blés les époux se bagarrent en plein champ et Marie termine "étendue au sol sans connaissance après avoir été poussée avec violence contre un mur et traînée par les cheveux à travers un grenier à blé ". Quelques mois plus tard, en public, " il la traite de gueuse, de putain, qu'elle avait été la sienne à sa sollicitation, il dit publiquement que l'enfant qu'elle avait eu pendant le mariage n'était point de lui mais d'une opération ecclésiastique puis la frappe au visage ». Un autre jour, il sort dans la rue et « lui inflige plusieurs coups de fouet avec les menaces les plus outrageantes et qu'il aurait effectué si des voisins charitables ne l'en avaient empêché, à ce moment il ajouta qu'il ne dépendait que de lui de la tuer ${ }^{18}$ ". L'ensemble des formes de violences conjugales est présent : brutalités physiques en public, injures, diffamation, accusation d'adultère et de crime sacrilège. Mais la déposition retranscrite ne rapporte rien de plus que les faits.

Cette faible présence des émotions dans les procédures de séparation pourrait certes s'expliquer par la pudeur verbale des déposants; cependant elle découle également du fonctionnement judiciaire et des coutumes, qui ne s'y prêtent guère, ainsi que nous l'avons exposé précédemment. Quelques indices issus d'une source précieuse, les factums, suggèrent qu'il

17. Arch. dép. des Deux-Sèvres, B-241 : marquisat de Fors. Sentences rendues par le sénéchal, 1702-1717.

18. Arch. dép. de la Vienne, 9B-504 : papiers du Roi (1767-1788). 
ne s'agit pas des seules explications possibles. Il convient dès lors de porter le regard sur les catégories sociales les plus favorisées.

En 1782, le procureur au siège royal d'Angers Pierre Delaunay présente un plaidoyer dans lequel il défend la cause de Marie-Henriette Françoise de Boscals de Reals, demanderesse en séparation contre son mari Charles Henri Bardel des Gléreaux, chef d'escadre breton de la Marine royale. Après avoir exposé les violences physiques et les multiples vexations infligées à l'épouse au cours de leur première année de mariage, le procureur s'appuie sur le Traité du Mariage du juriste Pothier. Celui-ci, dans son troisième chapitre, affirme que

« la Justice se décide à venir au secours d'une femme, elle n'exige pas aussi, pour obtenir la séparation des corps, que les sévices du mari aient été rendus tels que la femme ait eu à trembler pour ses jours. Il ne faut même pas qu'elle ait été frappée par son mari, si ce n'est dans la classe du peuple $[\ldots]^{19}$ ".

À Poitiers, quatre ans plus tard, l'avocat de Madeleine Allaire, fille d'un négociant de Saint-Domingue, en procédure contre son mari, Michel Creuzé, greffier en chef du bureau des finances de la généralité, s'avance plus loin encore dans la différenciation sociale des violences conjugales. L'épouse se dit victime d'insultes et de bousculades, qui constituent " des faits qui sont impuissants pour séparer des époux de la lie du peuple mais prennent entre personnes d'un état plus relevé un caractère de gravité qui peut devenir un moyen légitime de séparation ". Plus loin dans son mémoire, l'avocat entre plus avant dans sa description de ces femmes supposées frustes des catégories modestes :

" Accoutumées dès l'enfance à un langage grossier, les propos les plus outrageants les trouvent presque toujours insensibles; les emportements d'un mari brutal ne laissent aucune trace de ressentiment dans le cœur d'une femme et le calme le plus profond succède toujours à ces orages passagers $^{20}$."

La rhétorique de ces hommes de loi vise à comparer les comportements des maris, issus de la noblesse d'épée pour le premier et de la bourgeoisie de robe pour le second, à ceux des paysans et des petits artisans. Reflet de la société d'ordres de l'Ancien Régime, elle véhicule l'image de la femme du peuple soumise, indifférente aux violences, amnésique, insensible en raison de son manque d'éducation qui lui permet d'afficher un seuil de tolérance presque illimitée face aux brutalités de son mari. Ces préjugés

19. Quoique hors de notre champ géographique, les documents de cette procédure se trouvent aux Arch. dép. de la Vienne, F-20 : mémoire concernant la séparation des époux Bardel, fol. 10.

20. Arch. dép. de la Vienne, F-21 : 6 mémoires et réponses dans l'affaire de la dame Allaire contre son mari Creuzé-Dufrêne, mémoire $n^{\circ}$ 1, fol. 15. Merci à Laurent MASTORGIO, auteur d'une thèse de doctorat sur la famille Creuzé (soutenue à l'université de Poitiers en 2010) pour les informations communiquées sur ce couple. Voir son ouvrage Les Creuzé et leurs alliés. Châtellerault, 1600-1850, La Crèche, Geste éditions, 2018, 294 p. 
sociaux, constatés lors d'une précédente recherche ${ }^{21}$, méritent une étude affinée : les femmes de commerçants, d'artisans, de journaliers ne sont pas absentes des procédures de séparation et contredisent cette représentation. L'opinion présentée ci-dessus des deux avocats reflète plutôt la réalité des pratiques judiciaires. En effet, si les époux et épouses issus de la noblesse, de robe ou d'épée, ne constituent que $10 \%$ des 78 femmes et des 55 hommes qui utilisent le registre émotionnel dans leurs dépositions, ils représentent les deux tiers des 135 occurrences recensées. Peu nombreux, ils surutilisent ce lexique en comparaison des autres catégories sociales.

\section{Le registre émotionnel dans les procédures de séparation}

La lexicographie des émotions dans les actes de séparations conjugales offre un panel très diversifié. Elles sont presque toutes liées à l'évocation de violences maritales, à l'exception de trois cas de violences féminines sur les époux $^{22}$. Le recensement exhaustif des dépositions aboutit à 37 mots différents utilisés parmi les 135 mentions qui peuvent être rattachées au registre émotionnel. Les récurrences sont les suivantes : la fureur (17 mentions) et la colère (16) du mari, la peur de l'épouse (17) mais aussi la tendresse et le plaisir (11) que leur procurait leur vie commune à ses débuts.

Les termes utilisés se confondent parfois dans le sens qui leur est prêté : ainsi en va-t-il de la fureur et de la colère de certains époux. En mars 1787 est jugée en appel au présidial de Poitiers la procédure opposant les époux Chartier du Langis, installés à Parthenay. Le mari a abandonné le domicile depuis cinq ans, mais " revient de temps en temps l'insulter et la battre". En mars 1786, suite à une demande du mari de placer de force son épouse au couvent de l'Union chrétienne, elle lui propose une réconciliation. Le 12 avril, ils se rencontrent dans une auberge de Parthenay. Selon la déposition de l'épouse, les tenancières durent " faire appel à la garde de la ville pour emmener de force le sieur Guillon devant tout le public assemblé des habitants après qu'il l'ait accusé d'être une fiefée coquine, salope, gueuse, elle mérite d'être enfermée pour toujours dans une basse-fosse [...] il se jeta violemment sur elle et lui donne un coup de poing sur la figure qui lui fait saigner la bouche, il aurait continué ses violences si plusieurs personnes intervinrent heureusement et le forcèrent à se retirer lorsque les gardes arrivèrent, sans quoi on ne sait à quel point il aurait porté sa fureur ". Dix ans plus tôt, à Beaupréau ${ }^{23}$, Marie Thibaudeau tente d'obtenir la séparation de biens et de corps de son époux, Marc Richard, officier dans

21. "Clameur publique et violences conjugales au XVIII ${ }^{\mathrm{e}}$ siècle " dans CHAUvAuD, Frédéric et PrÉTou, Pierre (dir.), Clameur publique..., op. cit., p. 75-86.

22. Sur ce sujet, voir RÉGINA, Christophe, La violence des femmes. Histoire d'un tabou social, Paris, Max Milo Éditions, 2011, 318 p.; DAUPHIN, Cécile et FARGE, Arlette, De la violence et des femmes, Paris, Albin Michel, 1997, 208 p.; thèse de doctorat en cours de Fuchs, Charlotte, "Quand la poule chante devant le coq" : réalités et représentations de la " mégère » dans la France de la Renaissance, université de Tours (dir. P. Brioist).

23. Dans le sud du Maine-et-Loire, mais dans la juridiction du présidial de Poitiers. 
un régiment. Elle explique que, devant les soldats, il l'accuse de coucher avec un domestique puis " la fit entrer dans une chambre, lui donna des coups partout où il put malgré qu'elle fut grosse, qu'il lui envoya des coups de pieds dans le ventre [...] qu'il l'accuse d'avoir voulu l'empoisonner deux fois différentes [...] qu'un jour d'avril 1776 il lui donna un soufflet parce qu'elle avait mangé de l'ail et qu'au même moment lui arracha brusquement des mains un bas qu'elle achevait pour l'usage de son mari et le jeta au feu, que ces deux actions furent accompagnées de mouvements de colères, regards menaçants et jurements exécrables qui la forcèrent d'abandonner la maison et de se rendre à Fontenay $[. . .]^{24}$ ". Le vocable " colère ", au-delà d'un sentiment haineux, accentue l'impression rendue par cette déposition des risques physiques graves encourus par l'épouse.

Le terme de " peur ", pour sa part, ne s'avère pas le simple miroir de la fureur et de la colère masculines. Il peut également suggérer la proximité de la misère matérielle qui serait à l'origine de la demande de séparation, offrant une approche plus classique de cette dernière. Marie Petit, qui souhaite dissoudre la communauté qu'elle partage avec un laboureur de Mauzé en 1784, après seize années de mariage, explicite cette crainte du dénuement : " sa mère, Jeanne Roux, l'a dotée de 400 livres mais la dissipation, l'inconduite et le libertinage de son mari font qu'elle a peur de rester dans la plus cuisante misère, le jeu, l'ivrognerie, la fainéantise, les faux marchés, les entreprises irréfléchies lu ont déjà occasionné des frais immenses ${ }^{25}$ ". Sa peur s'accorde avec le désespoir que lui cause " journellement les incursions d'une foule d'huissiers porteurs de sentences contre son mari".

Le vocabulaire de la tendresse et de l'affection semble, pour sa part, socialement marqué : à une exception près, il est l'apanage des couples fortunés, à l'image du factum publié par Madeleine Allaire en 1787, à Poitiers, pour expliquer les motifs de sa demande de séparation. Retraçant la longue vie conjugale qu'elle vient de partager avec son époux, elle narre une de leurs nombreuses réconciliations, seize ans plus tôt, en recopiant une lettre qu'elle lui avait alors adressée : "J'ai reçu ta lettre aujourd'hui, cher ami, elle m'a fait répandre des larmes de joie et de tendresse. Je suis bien aise de te savoir arrivé en bonne santé; conserve-la toujours, cette santé qui m'est si précieuse, mon cher petit mari $[\ldots]^{26} . "$

Malgré ces tendances, il convient de souligner la variété des termes. Les descriptions émotives de la violence du mari constituent un tiers du corpus lexical, présentée comme animée par le désir de vengeance, la haine, la méchanceté ou la cruauté. Dans les mêmes proportions s'exprime la peur de l'épouse : la tristesse, les tourments, l'inquiétude, l'antipathie, l'incompatibilité, la honte, la douleur, le désespoir, le dégoût ou l'indignation sont utilisés afin de décrire celle-ci. Enfin, nous pouvons regrouper les occur-

24. Deux affaires issues des Arch. dép. de la Vienne, 9B-504 : papiers du Roi, 1767-1788.

25. Arch. dép. des Deux-Sèvres, 9B-209 : duché-pairie de Thouars, affaires civiles, 1784.

26. Arch. dép. de la Vienne, F-21 : mémoire nº 2, pour la dame Allaire, contre le sieur Creuzé-Dufrêne, 1787, fol. 7. 
rences restantes en deux catégories : le mépris affiché de l'homme (8\% des termes comme l'humiliation, l'indifférence, l'amertume, le mécontentement) et l'affection des époux. Celle-ci rassemble le quart des mots inclus dans ce lexique, utilisant les vocables divers comme la tendresse, le plaisir, la générosité, la passion, le bonheur, la joie ou l'espoir.

\section{Un vocabulaire normé mais non sexué}

L'identité des utilisateurs de ce lexique et la nature des sources judiciaires qui les recensent offrent quelques pistes de réflexion sur les usages de ces termes et leur éventuelle instrumentalisation. Dans ces procédures conçues pour protéger les biens des épouses, dont elles ont l'initiative quasi-exclusive, elles apparaissent logiquement comme les principales utilisatrices de ce vocabulaire ${ }^{27}$. Près de la moitié des occurrences repérées sont issues de leurs dépositions, tandis qu'un tiers provient de celles des témoins qui comparaissent, très majoritairement, en soutien de leur demande de séparation. Chaque catégorie se "spécialise": les femmes décrivent essentiellement leurs émotions propres, tandis que les témoins insistent sur les accès de colère, de haine ou de cruauté des époux. Cette pratique judiciaire semble intégrée dans les 48 affaires recensées, au cours desquelles les maris, peu présents, insistent essentiellement sur le caractère " épouvantable " de l'épouse ou sur son " inimitié ", voire sur un adultère supposé ${ }^{28}$. Les séparations plus retentissantes, comme celle des époux Creuzé qui s'étale sur la place publique de Poitiers en 1786 et 1787 à travers six factums ou celle, semblable, des époux angevins Bardel des Gléreaux, ainsi que deux procès en impuissance relevant de la Cour métropolitaine de Bordeaux, offrent une utilisation intensive du registre émotionnel, réparti de manière moins codifiée ${ }^{29}$. Les rôles s'inversent parfois. Michel Creuzé, dans son second mémoire en 1787, refuse la séparation et explique sa tristesse en des termes explicites : " J'aurais vu de sang-froid ma fortune en danger, je viens de m'exposer à bien des hasards pour mon épouse et ma belle-sœur : mais me sentir blessé dans tout ce que les hommes ont de plus cher, l'honneur et la vertu, par les mains d'une femme que j'ai toujours chéri, de la mère de mes enfants ${ }^{30}$ !"

Quelques années plus tôt, en 1782, l'avocat de Charles Bardel utilise une tactique similaire, mêlant l'affection et les regrets pour les violences

27. Voir la thèse sur les séparations de biens dans la haute noblesse parisienne de LANDELLE, Marie, “Les plaintes en séparation... ”, op. cit.

28. À ce propos, voir notre contribution "Les stratégies de défense masculines dans les affaires de violences conjugales (France, $\mathrm{XVII}^{\mathrm{e}}$-XVIII ${ }^{\mathrm{e}}$ siècles) " dans : FAGGION, Lucien, RÉGinA, Christophe et RIBEMONT, Bernard (dir.), La culture judiciaire. Discours, représentations et usages de la justice du Moyen Âge à nos jours, Dijon, Éditions universitaires de Dijon, 2014, p. 23-39.

29. Voir notre article : " Justice et virilité au XVIII ${ }^{\mathrm{e}}$ siècle. Les procès pour impuissance à Poitiers ", Revue Historique du Centre-Ouest, t. XI, 1 ${ }^{\mathrm{er}}$ sem. 2012, p. 142-162.

30. Arch. dép. de la Vienne, F-21 : mémoire no 2 , pour le sieur Creuzé-Dufrêne contre la dame Allaire, 1787, fol. 38. 
physiques infligées à Marie de Boscals, son épouse. Il explique que, " voulant lui donner des preuves authentiques du regret sincère qu'il a d'avoir offensé une femme aussi aimable que respectable, celle que son cœur a choisi, la seule qui put faire son bonheur, celle enfin qu'il aime et honore, il s'engage sur son honneur de la laisser absolument la maîtresse de son sort, s'en remet sous l'autorité paternelle [...]. Qu'elle vive tranquille elle qu'il aime plus que sa vie, oubliez ses excès, il les déteste $[\ldots]^{31}$ ". Les procès en impuissance, qui atteignent les hommes de manière singulière en mettant en cause leur virilité et leur identité masculine, permettent aux maris d'user du registre émotionnel de manière plus large que lors des simples procédures de séparations de biens. La " grande époque " de ces procès semble passée, depuis l'interdiction de l'épreuve dite du Congrès par le parlement de Paris en 1677. Ils s'étaient multipliés au XVII ${ }^{\mathrm{e}}$ siècle, suscitant de vives critiques en raison de l'impudicité des procédures établies par l'Église catholique afin de "vérifier " la virilité masculine. En effet, après un interrogatoire méticuleux des époux et l'examen des parties intimes de l'homme, le Congrès consistait en l'observation par un aéropage de juges, de médecins, de matrones et d'ecclésiastiques, de l'accomplissement de l'acte sexuel entre un mari soupçonné d'impuissance et son épouse. Il s'avère peu probant lors de plusieurs procès retentissants. Lorsque le marquis de Langeay échoue à cette épreuve en 1659, puis voit son mariage annulé, avant de convoler à nouveau en juste noces et de devenir père de trois filles, cette procédure semble définitivement disqualifiée ${ }^{32}$.

Toutefois, si elle disparaît, la possibilité de demander l'annulation d'un mariage pour raison d'impuissance demeure. La méthode se révèle plus discrète, puisqu'elle consiste désormais en l'inspection des organes génitaux de l'homme, sur lesquels des médecins patentés doivent exprimer leur avis quant à leur virilité ${ }^{33}$. Ainsi à Poitiers, au XVIII ${ }^{\mathrm{e}}$ siècle, cinq procès ont lieu dans la cité. L'un des plus marquants, selon Pierre Darmon, fut celui du marquis des Brosses; il trouva son épilogue en 1759 devant la Cour métropolitaine de Bordeaux installée à Poitiers ${ }^{34}$. À propos de son client, victime de deux procès en impuissance intentés par deux épouses successives, emprisonné au château d'Angoulême à plusieurs reprises, dépouillé de ses biens par son frère et sa sœur, son avocat, maître Loyseau, explique qu'il ressent " une profonde douleur, un malheur et une honte chaque jour plus grands [...] une pudeur détruite par l'inimitié de son frère et la haine que

31. Arch. dép. de la Vienne, F-20 : plaidoyer prononcé par maître Delaunay l'aîné, avocat au présidial d'Angers dans la cause de dame Marie-Henriette Françoise de Boscals de Reals contre messire Charles Henri Jacques Bardel des Gléreaux, 1782, fol. 45.

32. Voir DARMON, Pierre, Le tribunal de l'impuissance. Virilité et défaillances conjugales dans l'ancienne France, Paris, Seuil, 1979, 311 p.

33. Voir notre article mentionné infra (note 29) consacré à ces cinq procédures. Sur la virilité, voir les pages consacrées à son lien avec la définition de l'impuissance par Arlette Farge dans : CoRBIn, Alain, Courtine, Jean-Jacques, Vigarello, Georges (dir.), Histoire de la virilité, t. 1 : L'invention de la virilité. De l'Antiquité aux Lumières, Paris, Points Histoire, éd. 2015, p. 443-450.

34. DARmon, Pierre, Le tribunal de l'impuissance..., op. cit., p. 98. 
sa femme ajoute [...] il n'éprouve à son égard que le dégoût d'un mari trahi $[\ldots]^{35}$ ". À l'inverse, les épouses s'avèrent plus offensives dans les factums que les minutes des justices civiles ne peuvent le laisser transparaître. En effet, Madeleine Allaire, épouse de Michel Creuzé, ne se contente pas de nommer ses émotions mais porte des accusations contradictoires en accusant son mari d'être sujet à des " accès de rage et de cruauté " autant que d'avoir " un cour dur dénué de tout sentiment ${ }^{36}$ ".

Ce vocabulaire sensible dans les factums, comparé à son usage devant les justices civiles, offre une catégorisation qui ne peut être sexuée. Il infirme le constat d'un registre émotionnel systématique avec d'une part le masculin viril (la fureur, la vengeance, la colère, la haine...) et de l'autre, en reflet, le féminin fragile (la douleur, la honte, le désespoir).

L'existence, d'un lexique commun important autour de la tendresse et de l'affection, souligné plus haut ( $22 \%$ des mentions) et l'inversion de l'usage des émotions dans les procès d'exception, entraîne un classement d'une nature plus classique, juridique : certains usages se rattachent au vocabulaire de l'accusation et d'autres à celui de la défense. Il s'agit d'inspirer la répulsion d'un côté, la compassion de l'autre. Ces tactiques varient peu, et la forte féminisation du registre de la peur et de la douleur semble un reflet d'une réalité déformée par la procédure de séparation elle-même, qui s'enclenche très majoritairement à la sollicitation des épouses. Les rares affaires de violences conjugales féminines abondent en ce sens, ainsi que Christophe Regina le prouve à partir des procédures marseillaises ${ }^{37}$.

En Poitou, les trois cas identifiés de maris maltraités démontrent cette absence de genre du registre émotionnel dans les affaires de séparation. Ainsi, sur l'île de Noirmoutier en 1746, un nommé Moreau déserte la maison avec ses trois filles afin de fuir " la dame de Bernon son épouse, emplie de haine, qui le maltraitait continuellement, l'injuriait, le frappait avec cruauté, passait ses journées dans de violentes colères qui lui laissait craindre un destin funeste $^{38}$ ". Ici, l'homme a peur, la femme est haineuse et cruelle. Les recherches de Victoria Vanneau sur le XIX ${ }^{\mathrm{e}}$ siècle laissent supposer que ces cas, cachés car symboles d'un renversement des normes sexuelles, n'en demeurent pas moins réels ${ }^{39}$.

35. Arch. dép. de la Vienne, F-18 : mémoire pour le marquis des Brosses contre la dame de la Breuille de Chantresat, son épouse, 1759, fol. 4.

36 . Arch. dép. de la Vienne, F-21 : mémoire $n^{\circ}$ 2, pour le sieur Creuzé-Dufrêne contre la dame Allaire, 1787, fol. 40.

37. Voir son ouvrage cité en note 21.

38. Arch. dép. de la Vienne, 9B-503 : papiers du Roi, 1744-1766.

39. VANNEAU, Victoria, « Maris battus, femmes violentes : justice et société face à l'interversion des rôles conjugaux au XIX siècle " dans HAmmouche, A., 2012, p. 49-63 et La paix des ménages. Histoire des violences conjugales, XIXe-XXI siècles, Paris, Anamosa, 2015, 368 p. 


\section{Les émotions en dernier recours}

Par ailleurs, les quatre procès cités précédemment, dont la résonance déborde largement sur la place publique, rassemblent à eux seuls 54 des 135 termes " émotionnels " recensés à travers les factums, ce qui permet de s'interroger sur l'importance, évidente, des types de sources utilisées. Les représentants des institutions expriment peu leurs propres émotions : seuls un notaire, deux avocats et trois greffiers s'y adonnent en-dehors de la transcription des dépositions. Le notaire Charbonnier, de Mirebeau, enregistre en 1751 l'emprisonnement de François Gorin chez les cordeliers de Tours en raison des violences exercées sur sa femme et précise : "Sa fureur, sa violence et sa prodigalité inspirent la plus grande peur dans la paroisse $^{40}$. " Le greffe du présidial de Poitiers note, à propos du chirurgien Aubin, lorsqu'en mai 1790 il est enfermé " en prison dans cette ville en raison des procédés inouïs et indignes qu'il avait exercés contre Jeanne Vrignault sa femme et encore pour d'autres raisons dont le ministère public a eu connaissance et que la pudeur nous abstient (sic) d'évoquer ici ${ }^{41}$ ".

Mais les notaires et le greffe criminel constituent des sources marginales dans les affaires de séparation et de violences conjugales, ce qui, en soi, peut expliquer qu'ils soient marqués par ces affaires lorsqu'elles doivent se régler devant eux. $47 \%$ des termes émotifs sont issus de la justice civile et $35 \%$ des factums évoqués précédemment. Ces deux sources majeures devancent aisément les tribunaux ecclésiastiques (10\%) et criminels (8\%). Les imprimés, dont l'objectif est lié à la défense de la réputation d'un mari fortuné ou d'une épouse dont les mœurs sont remises en cause, utilisent stratégiquement ce registre émotionnel. En revanche, une analyse fine des minutes des justices civiles permet d'en dégager un usage clair : tandis qu'en première instance, auprès des justices seigneuriales, des émotions n'apparaissent que dans $7 \%$ des affaires, la proportion double dans les procédures d'appel, auprès du présidial.

Deux explications peuvent être proposées à ce constat. D'une part, voyant leur demande de séparation de biens pour des raisons matérielles, rejetée en première instance, certaines femmes ajoutent à la faillite de l'époux et aux privations qui s'ensuivent sa violence supposée afin de s'attirer la bienveillance du juge. D'autre part, la sociologie de ces procédures au présidial s'avère plus resserrée que pour celles qui se déroulent dans les instances seigneuriales. Celles-ci, sorte de justices de proximité d'Ancien Régime, touchent les laboureurs et les journaliers, les petits artisans des villages. En revanche, le présidial n'accueille jamais les épouses de ces dernières. Les demandeuses sont des femmes fortunées, de la noblesse ou de la bourgeoisie marchande, ainsi que des épouses de commerçants et d'artisans installés dans les villes de la province pour lesquelles l'usage du lexique des affects apparaît comme plus familier.

40. Arch. dép. de la Vienne, E4/88-517 : minutes Charbonnier, 1751-1756.

41. Arch. dép. de la Vienne, 1B2-205 : présidial de Poitiers, greffe criminel, 1779-1790. 
Le dernier paramètre à envisager repose sur la répartition des émotions selon les motifs des demandes. Les situations de fortes tensions, en particulier les violences verbales et physiques, représentent près de $62 \%$ des dépositions dans lesquelles des émotions sont retranscrites par les hommes de loi. La déposition d'un témoin lors de la séparation entre Jeanne Thomasset et François Huet en 1747 offre un exemple significatif du registre utilisé dans ce contexte : l'homme " couve contre elle une haine implacable qu'il n'a cessé de lui dire journellement les paroles les plus dures et les injures les plus atroces jusqu'à l'accuser publiquement du crime d'adultère [...] que les deux enfants qui sont nés de leur mariage ne viennent que du commerce illicite et scandaleux qu'elle a eu avec des prêtres et autres, qu'il veut les rejeter de sa maison [...]. Il déchaîne sur elle sa haine et sa cruauté jusqu'au point d'attenter à sa vie et de commettre des excès que la pudeur ne permet point d'exprimer, qu'elle a été obligée plusieurs fois de sortir de sa maison pour éviter les coups sous lesquels elle était certaine d'expirer ${ }^{42}$ ". La précarité matérielle engendre moins de références émotives, l'obtention de la séparation étant juridiquement plus aisée. Dans 27 \% des affaires recensées, elle en constitue le contexte, avec un lexique relevant généralement de la honte, de l'inquiétude ou de l'indignation. Ainsi la communauté de biens formée par Madeleine Piet et Jean Dieubon est-elle dissoute à Niort, en 1711, car " laditte Piet a souvent du chercher le sieur Dieubon au cabaret, où il était ivre et se répandait en injures et en débauches [...] elle éprouvait la honte de voir ainsi son mari oisif et empli de vin tout le jour $[. . .]^{43}$ ". L'ivrognerie ou le jeu ne sont pas les seules sources de la honte conjugale : les " mauvaises affaires " ou " mauvais marchés" qui désignent les commerçants en faillite sont également évoqués.

Les recherches menées sur les séparations et les violences de couples à l'époque moderne ont déjà noté la présence des émotions dans les dépositions et les narrations des désordres conjugaux. Scarlett BeauvaletBoutouyrie, en particulier, recense de nombreuses femmes de la haute noblesse exprimant leur amour, leur tristesse face à la furie des époux, leurs regrets et leurs tourments. Madame de Sévigné, Elisabeth Vigée Le Brun, la princesse des Ursins, la marquise du Châtelet ou celle de La Tour du Pin, Hortense et Marie Mancini laissent des mémoires ou des correspondances qui exposent en détail leurs démêlés avec des époux indélicats ${ }^{44}$. L'objet de nos recherches réside en l'étude de ces différends au niveau de femmes issues de catégories sociales plus modestes, petite noblesse provinciale, bourgeoisie marchande, commerçants, artisans ou laboureurs des campagnes poitevines. En dépit d'un fonctionnement judiciaire très normatif,

42. Arch. dép. de la Vienne, 1B2-205 : présidial de Poitiers, greffe criminel, 1779-1790.

43. Arch. dép. des Deux-Sèvres, 3B-52 : siège royal de Niort, séparations de biens, 17111776 .

44. Beauvalet-Boutouyrie, Scarlett, La solitude..., op. cit., p. 69-102. 
arasant sur le plan de la retranscription des émotions, les occurrences repérées ouvrent quelques interprétations suggestives, en particulier sur leur instrumentalisation afin d'obtenir gain de cause et une probable absence de catégorisation de genre en ce domaine.

\section{RÉSUMÉ}

Dans le Poitou, entre le milieu du $\mathrm{XvII}^{\mathrm{e}}$ siècle et le début de la Révolution française, près d'un millier de femmes et une dizaine d'hommes entament des procédures en séparation conjugale, de biens ou de corps. Si les difficultés matérielles expliquent le plus souvent leurs démarches, les archives des justices d'Ancien régime rapportent régulièrement les descriptions détaillées de vies de couple chaotiques, voire violentes. Parmi toutes les lectures possibles des dépositions, l'analyse du registre émotionnel est intéressant. Celui-ci s'avère freiné, en premier lieu, par une législation qui ne reconnaît que la mise en danger des biens de l'épouse comme motif valable de dissolution de la communauté, et en second lieu par les préjugés sociaux des hommes de loi qui se représentent les femmes " du peuple " comme insensibles aux violences maritales. Toutefois, à 135 reprises, les émotions ressenties sont mises en avant par les protagonistes, plaignantes ou témoins. Un recensement et une étude détaillée de ce lexique des affects permettent, particulièrement grâce à trois affaires de violences féminines, de mettre en lumière l'usage d'un vocabulaire non sexué, la relation de genre étant supplantée par le rapport accusé/victime. Enfin, les émotions, si elles décrivent des situations réelles, sont instrumentalisées et appelées en dernier recours, dans les procédures d'appel ou lorsque le scandale s'étale sur la place publique.

\section{ABSTRACT}

In Poitou, between the middle of the seventeenth century and the beginning of the French Revolution, nearly a thousand women and a dozen men initiated proceedings in conjugal separations. If material difficulties most often explain their decision, the archives of Ancien Régime justices regularly reported detailed descriptions of chaotic, even violent, married lives. The analysis the emotions expressed in the depositions is particularly worthy of attention. It is, however, hampered by legislation that only recognised the endangerment of the wife's property as a valid reason for dissolving the marriage, as well as by the social prejudices of lawyers who imagined the "women of the people" as being insensitive to marital violence. However, on 135 occasions, the emotions felt by the protagonists, complainants or witnesses were recorded. A census and a detailed study of this affective lexicon, especially through three cases of female violence, highlight the use of asexual vocabulary, the gender relationship being supplanted by the accused/victim relationship. Finally, emotions, if they described real situations, were instrumentalised and used as a last resort, in appeals procedures or when a scandal spread out into the public square. 